\title{
How to Avoid Overharvesting During Repeat Follicular Unit Excision Sessions by Calculating the Number and Density of Previously Excised Grafts
}

\author{
Kuniyoshi Yagyu, MD, FISHRS I Tokyo, Japan I nhttkyo11@kioicho.jp
}

\section{INTRODUCTION AND OBJECTIVE}

Donor area alopecia has become an issue after follicular unit excision (FUE). Even though the size of each FUE scar is small, a large number of scars can result in thinning of the hair in the donor area. ${ }^{1-5}$ In order to reduce thinning in the donor area, overharvesting of follicular units (FUs) should be avoided.

Sometimes after FUE at another clinic, a patient visits our clinic for a second session. To avoid overharvesting of FU grafts, it is necessary to know the total number of excisions, excision density, and hair follicle transection rate that occurred during the patient's previous session. However, it is usually difficult to obtain precise information about the graft transection rate from other clinics. In response to this concern, this author developed methods to calculate the excision density from previous surgery and to determine the safe excision density for future sessions.

\section{PATIENTS AND METHODS}

This study included five patients who visited our clinic for a second session of FUE. Each patient had undergone his first FUE hair transplantation in the frontal area at another clinic. Transplantation was carried out using a motorized FUE machine, a suction-assisted motorized FUE machine, or a robotic FUE machine. For their second session at our clinic, all patients requested strip excision surgery (FUT) to increase density in the frontal area.

Hair in the safe donor area was clipped before the FUT session. The number of FUs per square centimeter was counted using Vario Plus (Eschenbach) at three points: the parietal, the mastoid, and the mid-occipital areas. The average FU density at each point was measured in areas with and without FUE scars.

The baseline FU density in the area without FUE scars and the residual FU density in the post-FUE area were compared. The difference of the data gave us information about the excision or scar density. By measuring the surface area of FUE and multiplying it by the excision density, we then can calculate the number of excisions.

If we know (and can rely on) the number of grafts reported to have been transplanted, we can then estimate the percentage of "lost grafts" during the previous FUE harvesting, which we consider equal to the total transection rate.

\section{RESULTS}

\section{Ratio of FUs in the donor without hair regrowth (scars) to pre-op density}

The first patient, a 23-year-old male, had undergone 1,300 grafts at his first FUE session. His baseline FU density was 56 $\mathrm{FU} / \mathrm{cm}^{2}$ and his residual donor $\mathrm{FU}$ density was $43 \mathrm{FU} / \mathrm{cm}^{2}$. Therefore, his density of excisions was $56-43=13 \mathrm{FU} / \mathrm{cm}^{2}$. His ratio of scars to pre-op density was $13 / 56$, or $23 \%$.
The second patient, a 33-year-old male, had undergone 400 grafts at his first FUE session. His baseline FU density was $63 \mathrm{FU} / \mathrm{cm}^{2}$ and his residual donor FU density was 48 $\mathrm{FU} / \mathrm{cm}^{2}$. Therefore, his excision density was $15 \mathrm{FU} / \mathrm{cm}^{2}$ and his ratio of scars to pre-op density was 15/63, or $24 \%$.

The third patient, a 44-year-old male, had undergone 1,600 grafts at his first FUE session. His baseline FU density was $68 \mathrm{FU} / \mathrm{cm}^{2}$ and his residual donor FU density was 39 $\mathrm{FU} / \mathrm{cm}^{2}$. Therefore, his excision density was $29 \mathrm{FU} / \mathrm{cm}^{2}$ and ratio of scars to pre-op density was 29/68, or $43 \%$. (See Figure 1.)

The fourth case, a 21-year-old male, had undergone 720 grafts at his first FUE session. His baseline FU density was $62 \mathrm{FU} / \mathrm{cm}^{2}$ and his residual donor $\mathrm{FU}$ density was 35 FU/ $\mathrm{cm}^{2}$. Therefore, his

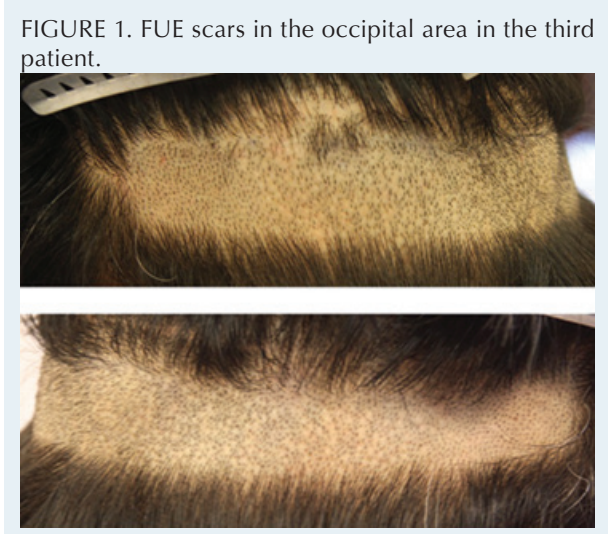

FIGURE 2. FUE scars in the occipital area in the fourth patient.

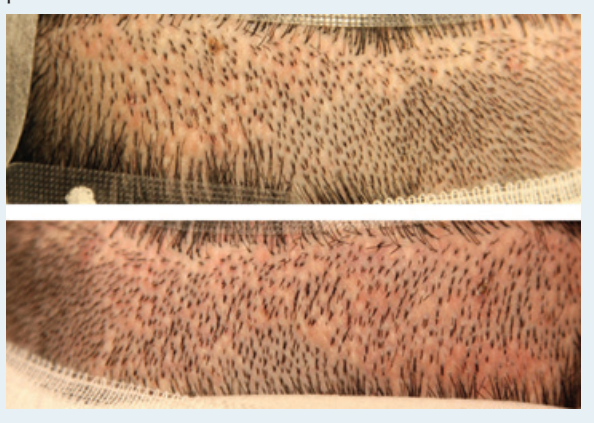

excision density was $27 \mathrm{FU} / \mathrm{cm}^{2}$ and ratio of scars to pre-op density was 27/62, or 44\%. (See Figure 2.)

The fifth patient, a 37-year-old male, had undergone 1,700 grafts at his first FUE session. His baseline FU density was 65 $\mathrm{FU} / \mathrm{cm}^{2}$ and his residual donor $\mathrm{FU}$ density was $40 \mathrm{FU} / \mathrm{cm}^{2}$. Therefore, his excision density was $25 \mathrm{FU} / \mathrm{cm}^{2}$ and ratio of scars to pre-op density was $25 / 65$, or $38 \%$.

\section{Total transection rate}

In the first case, the FUE surface area was $136 \mathrm{~cm}^{2}$. The estimated total number of excisions was $13 \times 136=1,768$. The availability of intact FUE grafts was 1,300/1,768, or $74 \%$. The total transection rate was $100-74=26 \%$.

In the second case, the FUE surface area was $48 \mathrm{~cm}^{2}$. The estimated total number of excisions was 720 . The availability of intact FUE grafts was $56 \%$. The total transection rate was $44 \%$.

In the third case, the FUE surface area was $100 \mathrm{~cm}^{2}$. The estimated total number of excisions was 2,900. The avail- 
ability of intact FUE grafts was 55\%. The total transection rate was $45 \%$.

In the fourth case, the FUE surface area was $80 \mathrm{~cm}^{2}$. The estimated total number of excisions was 2,160. The availability of intact FUE grafts was 33\%. The total transection rate was $67 \%$.

In the fifth case, the FUE surface area was $160 \mathrm{~cm}^{2}$. The estimated total number of excsions was 4,000. The availability of intact FUE grafts was $43 \%$. The total transection rate was $57 \%$.

\section{Cross section of the scalp tissue}

The donor strip was slivered during the FUT session. Cross sections of the donor scalp revealed that scar tissue occupied the full thickness of the scalp from the epidermis to the subcutaneous adipose tissue at each FUE excision. (See Figures 3, 4, and 5.)
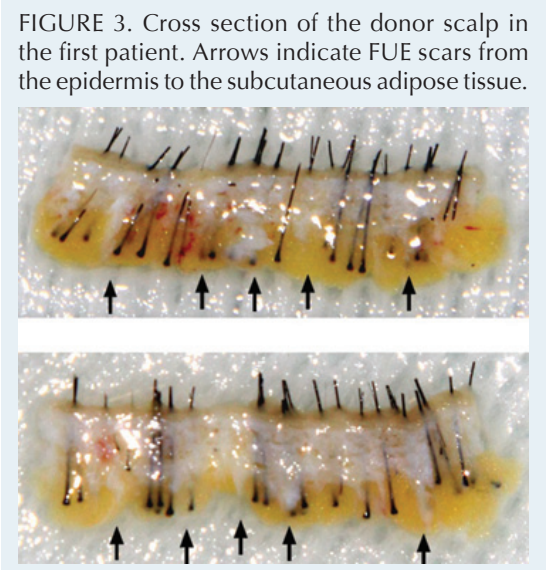

FIGURE 4. Cross section of the donor scalp in the fourth patient. Arrows indicate FUE scars.

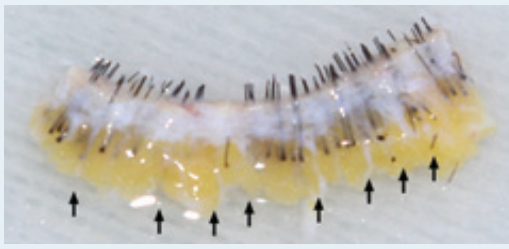

FIGURE 5. Cross section of the donor scalp in the fifth patient. Arrows indicate FUE scars.

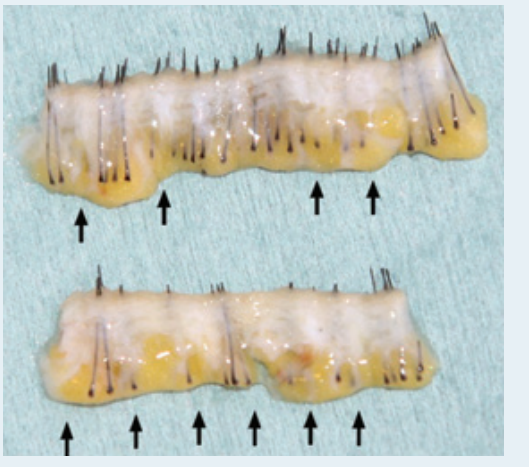

\section{DISCUSSION}

In repeat FUE cases, it is usually difficult to obtain precise information concerning excision density and graft transection rate that occurred at the first FUE surgery at a previous clinic. In order to avoid visible thinning in the donor area after subsequent sessions, we need to assess the safe excision density to maintain cosmetic residual density in the donor area.

Visible donor area scarring and low cosmetic density will occur if too many FUs are excised from the donor area. Patients tend to consider that it is the last doctor seen who is responsible for the visible thinning and donor area alopecia. The initial doctor, who harvested too many grafts at the first FUE session, is usually not accused as long as the patient does not notice the thousands of

FUE scars without hair regrowth in the donor area.

In order to avoid thinning of the donor after repeat FUE sessions, we need a guideline for safe excision limits during repeat sessions. The donor will look noticeably thin if more than $50 \%$ of the original terminal hair is harvested after one or more FUE sessions. We need to limit the total number of
FU grafts harvested to less than $50 \%$ of the baseline FU density. If cosmetic residual density, in general, should be more than $40 \mathrm{FU} / \mathrm{cm}^{2}$ in the donor area, ${ }^{5}$ the safe excision density will be less than $40 \%$ of the baseline density in a patient with a baseline density of $56-68 \mathrm{FU} / \mathrm{cm}^{2}$ in this study.

In repeat FUE cases, we should measure the ratio of residual density and baseline density before the session to calculate the excison density; that is, we need to determine what already has been harvested. Then, we can calculate the percentage of FUE excisions in the donor area, and we will be able to estimate the safe excision density for the next FUE session to avoid overharvesting. Other patient characteristics such as hair color and thickness and desired hair length should also be considered. It is also important to consider different FU densities and excision densities in various parts of the donor area.

Donor hair will be lost if hair follicles are transected during FUE. Lifetime donor availability is limited in hair transplantation. There are often not enough donor follicles to cover a wide area of hair thinning in a patient with type VI and VII male pattern hair loss.

We have to try to avoid transection during hair transplantation. Even if some percentage of hair follicle transection is inevitable during FUE, it is important to keep the follicle transection rate as low as possible during the procedure. If the given numbers of transplanted FUs were correct, then the calculated total transection rates in the patients studied were unacceptably high. Another reason for these high numbers of "lost grafts" could be partially transected FUs that were discarded or buried grafts.

It is not the purpose of this study to compare the quality of various kinds of FUE machines. When used properly, various FUE instruments have their own excellent quality. However, if a machine is not used properly, the hair follicle transection rate will increase.

If an FU graft is totally transected during FUE, the transected graft doesn't grow terminal hair after implantation in the recipient area or in the original donor area. This is the reason why it is important to minimize total graft transection and even follicle transection (partial graft transection) during FUE. ${ }^{6}$

The ratios of lost grafts mostly due to total graft transection were high in patients 3, 4, and 5. Many FU grafts were destroyed and lost during the first FUE sessions in these cases. This may have been due to poor technique, improper instrumentation, or difficult patient characteristics. Performing FUE in a test area in these patients may be a good idea. If the safe excision density should be less than $40-50 \%$ of the baseline density, further FUE graft harvesting would be unsuitable in the previously harvested area. Possibly, more FUE grafts can be harvested in other safe donor areas in future FUE sessions in these patients.

If one hair had regrown from a 2- to 3-hair FU graft, it was counted as a 1-hair FU and it was not counted as FU without hair regrowth in this study. This means that the real number of punch holes and the actual (total plus partial) graft transection rate may have been higher than the estimated total graft transection rate in this study. ${ }^{6}$

Usually, many of the 2- to 3-hair FUs with thick hair had already been harvested at the previous FUE session, and 
many of the residual FUs are 1- to 2-hair FUs with thin hair. This means that an excision density of $50 \%$ may be too high and a safe excision density will be less than $40 \%$ of the baseline density after repeat FUE sessions. ${ }^{5}$ If the patient wants to wear a short hairstyle in the future, the safe maximum excision density should be less than $40 \%$ of the baseline density.

FUE scars occupy the full thickness of the scalp from the epidermis to the subdermal adipose tissue, and thousands of FUE scars can be distributed in the occipital area. This means that nearly half of the occipital area could be transformed into full-thickness scar tissue after repeat FUE sessions. It is different from an FUT scar, which results in a full-thickness linear scar ideally 1-2mm wide, leaving most of the donor scalp intact without scar tissue.

\section{CONCLUSION}

In order to avoid overharvesting of FU grafts and thinning of hair in the donor area in repeat FUE cases, the physician needs to know how many FUs had already been harvested during the previous session. Depending on various factors, the calculation of the previous number of excisions, excision density, and rate of totally transected grafts will provide the physician with useful information to maintain a safe excision density during repeat FUE sessions.

\section{References}

1. True, R., et al. FUE roundtable. Hair Transplant Forum Int'l. 2016; 26(4):134-159.

2. Jimenez, F. Musicians as a source of inspiration for surgeons. Hair Transplant Forum Int'l. 2012; 22(2):41-44.

3. Knudsen, R, Ethics and follicular unit extraction. Hair Transplant Forum Int'l. 2016; 26(4):133.

4. Cole, J.P. Status of individual follicular group harvesting. Hair Transplant Forum Int'l. 2009; 19(1):20-24.

5. Keene, S., et al. Determining safe excision limits in FUE: Factors that affect, and a simple way to maintain, aesthetic donor density. Hair Transplant Forum Int'I. 2018; 28(1):1-11.

6. True, R. Graft quality index: a morphologic classification of follicular unit excision (FUE) grafts. Hair Transplant Forum Int'l. 2018; 28(2):45-53.

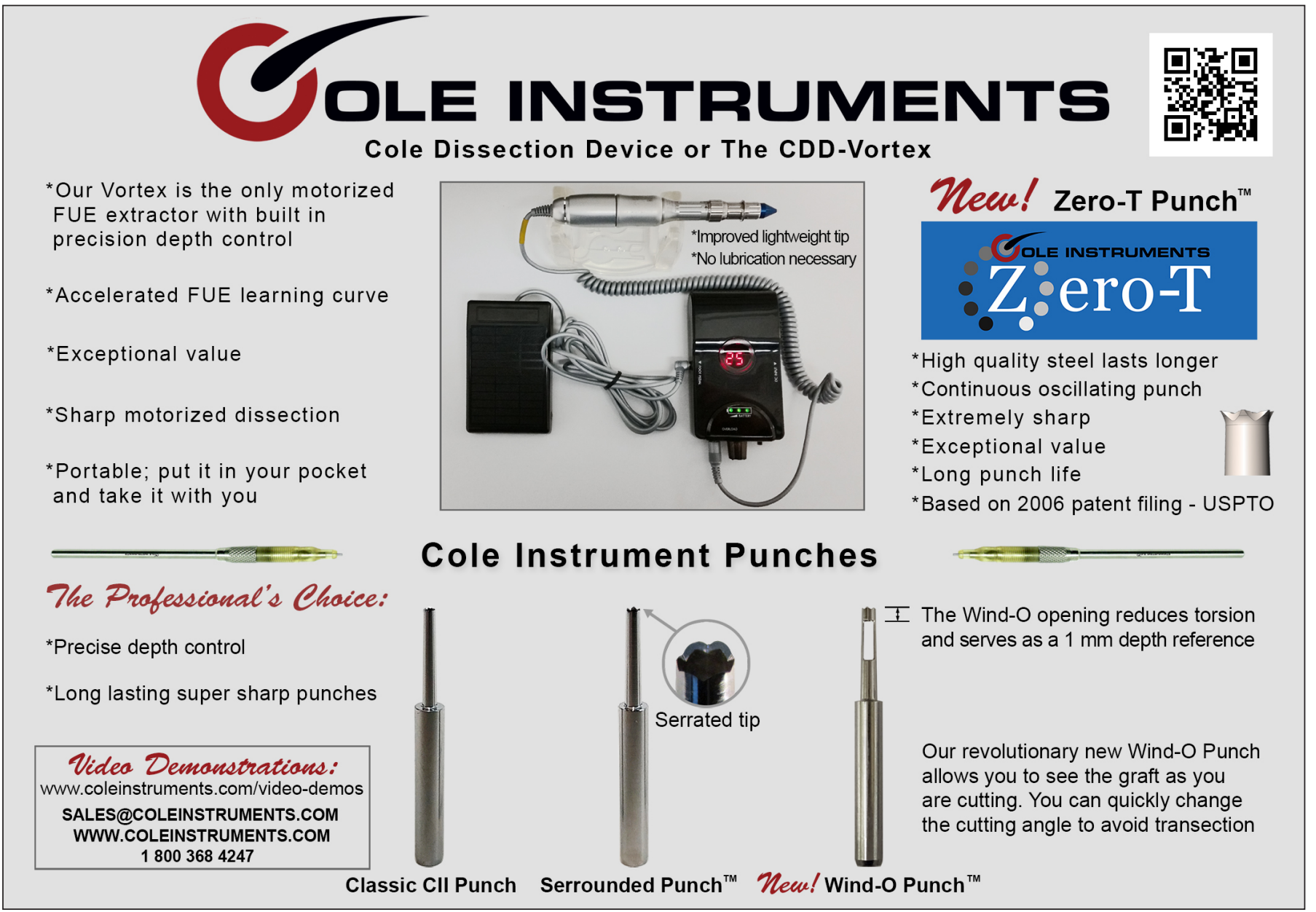

